\title{
Total motile sperm count: a better way to rate the severity of male factor infertility?
}

\author{
Edson Borges $\mathrm{Jr}^{1,2}$ \\ ${ }^{1}$ Fertility - Centro de Fertilização Assistida, São Paulo/SP, Brazil \\ ${ }^{2}$ Instituto Sapientiae - Centro de Estudos e Pesquisa em Reprodução Assistida, São Paulo/SP, Brazil
}

\begin{abstract}
Subfertility affects up to $18 \%$ of couples, and poor semen quality has been implicated in approximately half of the cases. Semen analysis has been universally used to assess semen quality. In 2010, the World Health Organization (WHO) defined new reference values for sperm parameters to discriminate between normal and abnormal semen samples (including oligozoospermia, asthenozoospermia, teratozoospermia, and the combinations of these factors) (Cooper et al., 2010). However, reports suggest that the prognostic relevance of the $2010 \mathrm{WHO}$ classification system is poor (Esteves et al., 2012; Hamilton et al., 2015).
\end{abstract}

Semen parameters such as volume, concentration, and motility can be pooled to yield a different manner to express sperm quality: the total motile sperm count (TMSC). The TMSC is obtained by multiplying the volume of the ejaculate by the sperm concentration by the proportion of progressive motile sperm divided by $100 \%$ (Ayala et al., 1996), and can be calculated from the neat semen (prewash TMSC) or after semen preparation (postwash TMSC).

Several reports have shown that the TMSC has a prognostic value in couples undergoing intrauterine insemination (van Weert et al., 2004; Badawy et al., 2009; Nikbakht \& Saharkhiz, 2011) in predicting total fertilization failure after conventional in vitro fertilization (IVF) (Rhemrev et al., 2001). Recently, the TMSC was shown to have a stronger correlation with the spontaneous ongoing pregnancy rate than the WHO 2010 classification system (Hamilton et al., 2015). Couples with a TMSC $<5 \times 10^{6}$ had a significantly lower chance of spontaneous ongoing pregnancy than couples with a TMSC $>5 \times 10^{6}$.

Hamilton et al. (2015) proposed three prognostic groups of semen quality according to the TMSC: (i) $<5$, (ii) between 5 and 20 , and (iii) $>20 \times 10^{6}$ spermatozoa (the latter being considered a normal TMSC value). Additionally, it was suggested that the TMSC should be the method of choice to rate male infertility. However, little is known about the prognostic value of the TMSC classification for ICSI outcomes.

In a study performed at our center with couples undergoing ICSI due to male factor infertility, the TMSC was the only seminal parameter to affect the formation of high-quality embryos on D2 (OR: 1.18, CI: 1.03-1.35, $P=0.013$ ) and D3 (OR: 1.12, CI: 1.07-1.29, $P=0.037$ ), the formation of blastocysts (OR: 1.16, CI: 1.04-1.26, $P=0.011$ ), and the blastocyst expansion grade (OR: 1.27, CI: $1.01-1.60, P=0.042$ ). The TMSC was also a better predictor for miscarriage (OR: $0.52, \mathrm{CI}: 0.28-0.90, P<0.045)$ than the WHO cutoff values. Additionally, significantly higher fertilization rates $(84.9 \pm 14.4$ vs. $81.1 \pm 15.8$, $P=0.016)$ and lower miscarriage rates (17.9\% vs. $29.5 \%$, $P=0.041$ ) were seen in couples with normal versus abnormal TMSC (unpublished results).

The reference values established by the WHO in 2010 were determined based on men who had recently fathered a child. Therefore, it is not wise to define fertility or infer- tility based on semen parameters below or within/above the WHO cutoffs. Moreover, the WHO classification system seems to be much more stringent than the TMSC when it comes to defining male infertility, since more couples have been diagnosed with male infertility when the WHO criteria were applied than when the TMSC was used. A pertinent explanation for this discrepancy is that the TMSC considers absolute sperm parameter values simultaneously, while the WHO criteria treats sperm parameters discretely.

ICSI is the treatment of choice for couples with male factor infertility, since it bypasses sperm limitations related to the fertilization process. Therefore, it has been suggested that seminal parameters do not impact the outcomes of ICSI. However, one might argue that male factor infertility, when rated by the TMSC, affects the outcome of ICSI, a situation that is not observed when the normality of seminal parameters is assessed by the WHO classification system.

Our results have shown that the TMSC is a better predictor for ICSI outcomes than the criteria recommended by the WHO. This new information supports the organization of further prospective randomized studies to verify the superiority of the TMSC in relation to the WHO classification system in rating male infertility severity.

\section{REFERENCES}

Ayala C, Steinberger E, Smith DP. The influence of semen analysis parameters on the fertility potential of infertile couples. J Androl. 1996;17:718-25.

Badawy A, Elnashar A, Eltotongy M. Effect of sperm morphology and number on success of intrauterine insemination. Fertil Steril. 2009;91:777-81.

Cooper TG, Noonan E, von Eckardstein S, Auger J, Baker HW, Behre HM, Haugen TB, Kruger T, Wang C, Mbizvo MT, Vogelsong KM. World Health Organization reference values for human semen characteristics. Hum Reprod Update. 2010;16:231-45.

Esteves SC, Zini A, Aziz N, Alvarez JG, Sabanegh ES Jr, Agarwal A. Critical appraisal of World Health Organization's new reference values for human semen characteristics and effect on diagnosis and treatment of subfertile men. Urology. $2012 ; 79: 16-22$.

Hamilton JA, Cissen M, Brandes M, Smeenk JM, de Bruin JP, Kremer JA, Nelen WL, Hamilton CJ. Total motile sperm count: a better indicator for the severity of male factor infertility than the WHO sperm classification system. Hum Reprod. 2015;30:1110-21.

Nikbakht R, Saharkhiz N. The influence of sperm morphology, total motile sperm count of semen and the number of motile sperm inseminated in sperm samples on the success of intrauterine insemination. Int $]$ Fertil Steril. 2011;5:168-73. 
Rhemrev JP, Lens JW, McDonnell J, Schoemaker J, Vermeiden JP. The postwash total progressively motile sperm cell count is a reliable predictor of total fertilization failure during in vitro fertilization treatment. Fertil Steril. 2001;76:884-91. van Weert JM, Repping S, Van Voorhis BJ, van der Veen F, Bossuyt PM, Mol BW. Performance of the postwash total motile sperm count as a predictor of pregnancy at the time of intrauterine insemination: a meta-analysis. Fertil Steril. 2004;82:612-20. 